\title{
Virtual gastroscopy for the evaluation of stomach malignancy
}

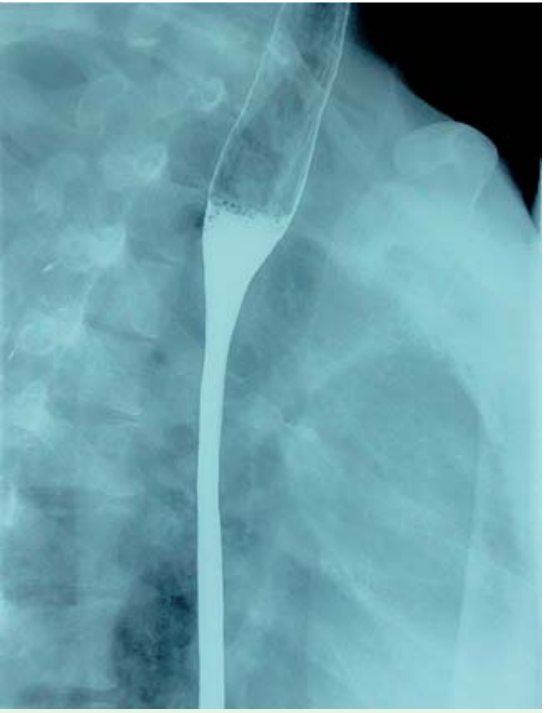

Fig. 1 Double-contrast total gastrointestinal examination showing a smooth narrowing of the esophagus between the level of the aortic arch and the cardia.

A 50-year-old woman presented with an 18-month history of nonprogressive dysphagia, early satiety, weight loss, and an aversion to greasy food. Physical examination revealed an old $20-\mathrm{cm}$ scar on her right thorax following treatment for breast carcinoma, for which she had undergone radical mastectomy and chemotherapy 7 years previously. Conventional endoscopy (EG-2990Zi, 11.4-mm diameter; Pentax, Tokyo, Japan) failed because of the narrowness of the esophagus. Subsequent double-contrast total gastrointestinal examination confirmed the narrowing of the esophagus between the level of the aortic arch and the cardia with a smooth internal surface ( $\bullet$ Fig. 1), as well as stomach stiffness. An ultraslim endoscope (EG-1540; Pentax) passed snugly through the narrowed area allowing a deep biopsy to be taken from the gastric body. Immunostaining of the biopsy gave the following results: estrogen receptor $(\mathrm{ER})+$; progesterone receptor $(\mathrm{PR})+$; carcinoma antigen (CA) 15-3+; cytokeratin (CK) 20-; and focal gross cystic disease fluid protein (GCDFP)+/-, consistent with metastatic linitis plastica from a primary breast carcinoma.

A subsequent computed tomography (CT) scan revealed a thickened stomach wall. No gas-producing powder was given prior to virtual gastroendoscopy. No ulcer or mass was detected in the stomach, and the gastric folds at the greater curvature were coarse but remained largely intact ( Fig. 2a). In this case, virtual gastroendoscopy without gas inflation showed nothing suggestive of linitis plastica.

A second patient with a confirmed Borrmann IV gastric carcinoma underwent conventional endoscopy and CT-based vir- tual gastroendoscopy prior to surgery. During conventional endoscope, the scope could not be retroflexed in the lower gastric body. This patient was given $3 \mathrm{~g}$ gasproducing power (Dongfeng Chemical Ltd, Qingdao, China) to generate approximately $360 \mathrm{~mL}$ carbon dioxide in the stomach and virtual gastroendoscopy demonstrated an obviously disfigured stomach. An obvious stricture noted in the distal gastric body after gas inflation could have been the explanation for the difficulties with the retroflexion maneuver during conventional endoscopy. Unlike the uninvolved antrum, the gastric body and fundus showed several characteristic large filling defects ( $\nabla$ Fig. $\mathbf{2}$ b,c). This was a result of uneven stiffness caused by the malignant infiltration of the gastric wall.

CT-based virtual gastroendoscopy has been considered as an alternative approach to examination of the upper gastrointestinal tract where there are substantial risks or difficulties with conventional endoscopy [1,2]. However, this application has seldom been reported, and none of the previous reports have demonstrated linitis plastica on virtual gastroendoscopy. Here we have presented two patients who underwent virtual gastroendoscopy evaluation, one because of the narrowness of the esophagus and the other because of a stricture within the stomach. Preparing patients with gasproducing powder is necessary to reveal the characteristic "ugly stomach" of linitis plastica. More studies on virtual gastroendoscopy are warranted to further elucidate its value in patients where conventional endomicroscopy is difficult.
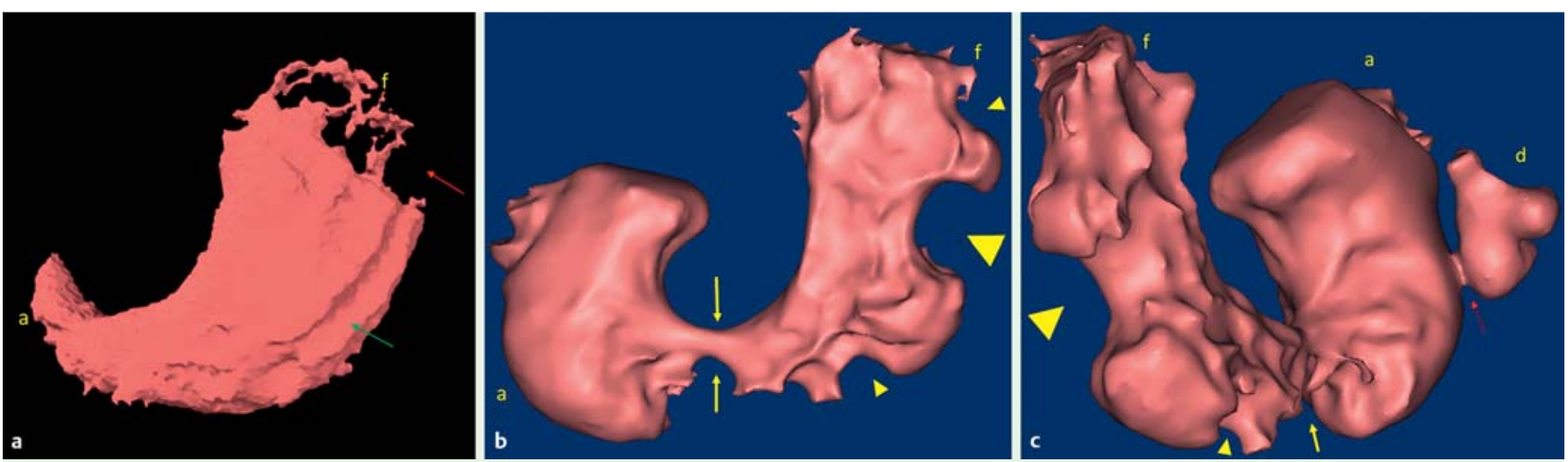

Fig. 2 Virtual gastroendoscopy views in two patients with gastric malignancy. a The gastric lumen from outside the stomach. No mass or ulcer is detected, but excessive food retention at the fundus (red arrow) and coarse gastric folds (green arrow) can be seen. b Anterior view of a Borrmann IV gastric carcinoma. Uneven stiffness caused by malignant infiltration of the gastric wall is producing many large filling defects (yellow arrow heads). A prominent stricture in the distal gastric body (yellow arrows) was probably the cause of the difficulty reflexing the conventional endoscope. c Posterior view of a Borrmann IV gastric carcinoma (red arrow) in the pylorus (f, gastric fundus; a, gastric antrum; d, duodenum). 
Endoscopy_UCTN_Code_CCL_1AB_2AH_3AC

Competing interests: None

\section{Ming Li, Xiu-Li Zuo, Yan-Qing Li}

Department of Gastroenterology, Shandong University Qilu Hospital, Jinan, China

\section{References}

1 Ogata I, Komohara Y, Yamashita $Y$ et al. CT evaluation of gastric lesions with three-dimensional display and interactive virtual endoscopy: comparison with conventional barium study and endoscopy. AJR Am J Roentgenol 1999; 172: $1263-1270$

2 Inamoto K, Kouzai K, Ueeda T et al. CT virtual endoscopy of the stomach: comparison study with gastric fiberscopy. Abdominal imaging 2005; 30: 473-479

\section{Bibliography}

DOI http://dx.doi.org/

10.1055/s-0034-1365813

Endoscopy 2014; 46: E320-E321

(c) Georg Thieme Verlag KG

Stuttgart · New York

ISSN 0013-726X

\section{Corresponding author}

\section{Xiu-Li Zuo, MD}

Department of Gastroenterology Shandong University, Qilu Hospital 107 Wenhuaxi Road Jinan 250012

China

Fax: +86-531-82166090

xiulizuo@gmail.com 